\title{
Laparoscopy-assisted micropercutaneous choledocholithotripsy with holmium laser in a cholecystectomized patient: an initial report
}

\author{
Kaan Gökçen ${ }^{1}$, Mustafa Atabey², Pınar Gökçen³ ${ }^{3}$ Gökhan Gökçe ${ }^{1}$ \\ ${ }^{1}$ Department of Urology, School of Medicine, Cumhuriyet University, Sivas, Turkey \\ ${ }^{2}$ Department of General Surgery, School of Medicine, Cumhuriyet University, Sivas, Turkey \\ ${ }^{3}$ Department of Gastroenterology, School of Medicine, Cumhuriyet University, Sivas, Turkey
}

Videosurgery Miniinv 2017; 12 (4): 443-447

DOI: https://doi.org/10.5114/wiitm.2017.72328

\begin{abstract}
We present a novel minimally invasive technique, laparoscopy-assisted micropercutaneous choledocholithotripsy, for choledocholithiasis that cannot be treated with other endoscopic techniques. This technique includes standard laparoscopic exploration of the common bile duct, combined with an all-seeing needle and holmium laser lithotripsy. As is known, an all-seeing needle is used in micropercutaneous nephrolithotomy for middle-sized renal stones. In this technique, an all-seeing needle was inserted into the dilatated common bile duct under laparoscopic vision and then a lithotripsy procedure was performed with a holmium laser behind the biliary stent. A cholecystectomized female patient with a 21- $\mathrm{mm}$ stone in the common bile duct who previously underwent an unsuccessful endoscopic retrograde cholangiopancreatography procedure was operated on in our service with laparoscopy-assisted micropercutaneous choledocholithotomy without a T-tube. This novel procedure was completed uneventfully and the patient was discharged without any complications. In the future, this procedure will hopefully be a treatment modality in choledocholithiasis that cannot be treated by other minimally invasive techniques.
\end{abstract}

Key words: laparoscopy, micropercutaneous, choledocholithotripsy.

\section{Introduction}

Choledocholithiasis is the second most common complication in patients with gallstones, and it is seen in approximately $5 \%$ of asymptomatic patients and in $10-15 \%$ of symptomatic patients [1]. Since laparoscopic cholecystectomy has become the gold standard in the removal of gallstones and has begun to be administered commonly, the rate of preference of laparoscopic approaches in the treatment of common bile duct stones has increased. Optimal treatment options of common bile duct stones has not been clearly revealed, and open bile duct exploration, laparoscopic bile duct exploration, endoscopic retrograde cholangiopancreatography (ERCP) combined with preoperative, intraoperative or postoperative laparoscopic cholecystectomy, and sphincterotomy are among the treatment methods that are being used. In the minimally invasive treatment of bile duct stones, methods such as saline lavage, fluoroscopy-guided basket administration, biliary balloon catheter, cystic channel dilatation, ampullary balloon dilatation, antegrade sphincterotomy, and the flexible endoscopic method have been described [2]. In addition to the minimally invasive methods mentioned above, holmium laser lithotripsy is also used in the treatment of complex bile duct stones accompanied with choledochoscope in case of a recent failed ERCP. Laser lithotripsy, which has been commonly used in the treatment of renal calculi, was primarily used in

\section{Address for correspondence}

Assist. Prof. Kaan Gökçen, Department of Urology, School of Medicine, Cumhuriyet University, Cumhuriyet University Hospital,

58010 Sivas, Turkey, phone: +90 346 258000, e-mail: kaangokcen@hotmail.com 


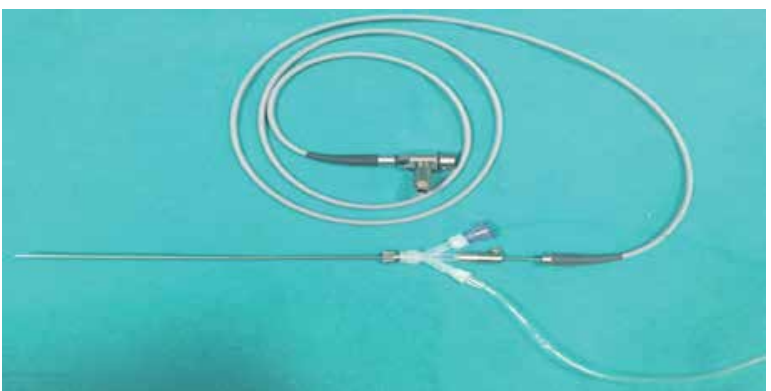

Photo 1. Image of micropercutaneous system includes 3-way connector, micro-optic, irrigation system and laser probe, and 4.8-Fr sheath

the treatment of biliary stones as presented by Orii et al. in 1983, and is now combined with laparoscopy in the treatment of common bile duct stones today [3].

In order to minimize the percutaneous nephrolithotomy ( $\mathrm{PNL}$ ) process that is the primary treatment option for renal calculi larger than $20 \mathrm{~mm}$, thanks to the technological improvements in renal stone treatment, the micropercutaneous nephrolithotomy (micro-PNL) method, which includes using a holmium laser together with an "all-seeing needle", has been developed [4]. The all-seeing needle is an instrument that includes a $0.9-\mathrm{mm}$ micro-optic with 10000 pixel resolution, and inner and outer puncture shafts. In the micro-PNL technique, a 4.8 Fr (16 Gauge) all-seeing needle (PolyDiagnost, Pfaffenhofen, Germany) was inserted into the selected renal calyx under direct vision or fluoroscopy. After insertion of the nee- dle into the calyceal system, the optical instrument and the inner sheath were removed and a 3-way connector was applied for insertion of the micro-optic, irrigation system and laser probe (Photo 1).

Here, we report an initial case of combination of laparoscopic common bile duct exploration (LCBDE) and micropercutaneous choledocholithotripsy in choledocholithiasis with failed ERCP.

\section{Case report}

A 60-year-old female patient referred to our service with the complaint of gradually increasing abdominal pain. In the physical examination of the patient, who had a history of laparoscopic cholecystectomy 2 years ago, type 2 diabetes mellitus and coronary artery disease, the skin and sclera were observed to be icteric. In the laboratory analyses, no feature was observed in the hemogram and coagulation examinations. In biochemical analyses, the parameters had the following values: glucose $210 \mathrm{mg} / \mathrm{dl}$ (74-106); aspartate aminotransferase (AST) $102 \mathrm{U} / \mathrm{l}$ (0-35); alanine aminotransferase (ALT) $180 \mathrm{U} / \mathrm{l}$ (0-35); $\gamma$-glutamyl transferase (GGT), $824 \mathrm{U} / \mathrm{l}$ (0-38); alkaline phosphatase (ALP) $361 \mathrm{U} / \mathrm{l}$ (30-120); total bilirubin $6.30 \mathrm{mg} / \mathrm{dl}(0.3-1.2)$; and direct bilirubin $3.08 \mathrm{mg} / \mathrm{dl}$ (0.0-0.2). Hepatitis serology and tumor markers were negative. In contrasted abdominal tomography and magnetic resonance (MR) cholangiography, intrahepatic biliary tracts were dilated; the
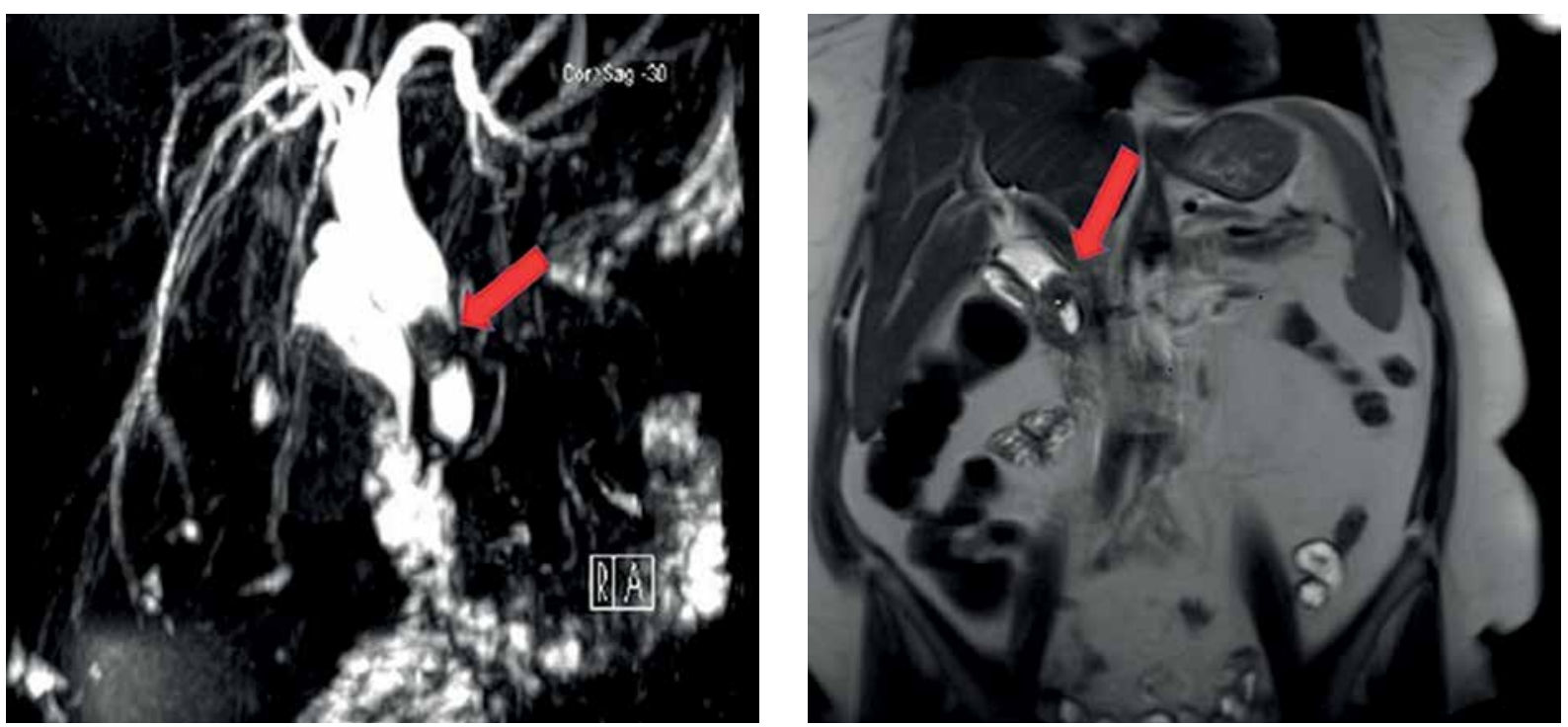

Photo 2. Representative images of magnetic resonance cholangiography and computed tomography scans demonstrating 21-mm-sized stone in middle-distal choledochal duct 
diameter of the common bile duct was measured as $18 \mathrm{~mm}$ at the widest location, and a stone with an approximately 21-mm diameter was observed in the middle-distal part of the lumen (Photo 2). Even though sphincterotomy was performed, our gastroenterology service upon the observation of a calculus across a segment of approximately $3-\mathrm{cm}$ in the common bile duct in ERCP inserted a biliary stent in the patient upon the failure to remove the calculi to the duodenum via a balloon. A pancreatic stent was inserted into the patient, who was administered $1 \mathrm{~g}$ of parenteral ceftriaxone twice a day, and $500 \mathrm{mg}$ of metronidazole twice a day for a week, immediately before the operation in order to prevent the development of pancreatitis.

The patient was accepted to the general surgery service after the failed ERCP, and the exploration of the primary common bile duct and laparoscopy-assisted micropercutaneous choledocholithotomy and holmium laser lithotripsy were performed after the approval of the Human Ethics Committee of our university and written approval of the patient. The patient was discharged 5 days after the surgery, following an uneventful postoperative period. One month
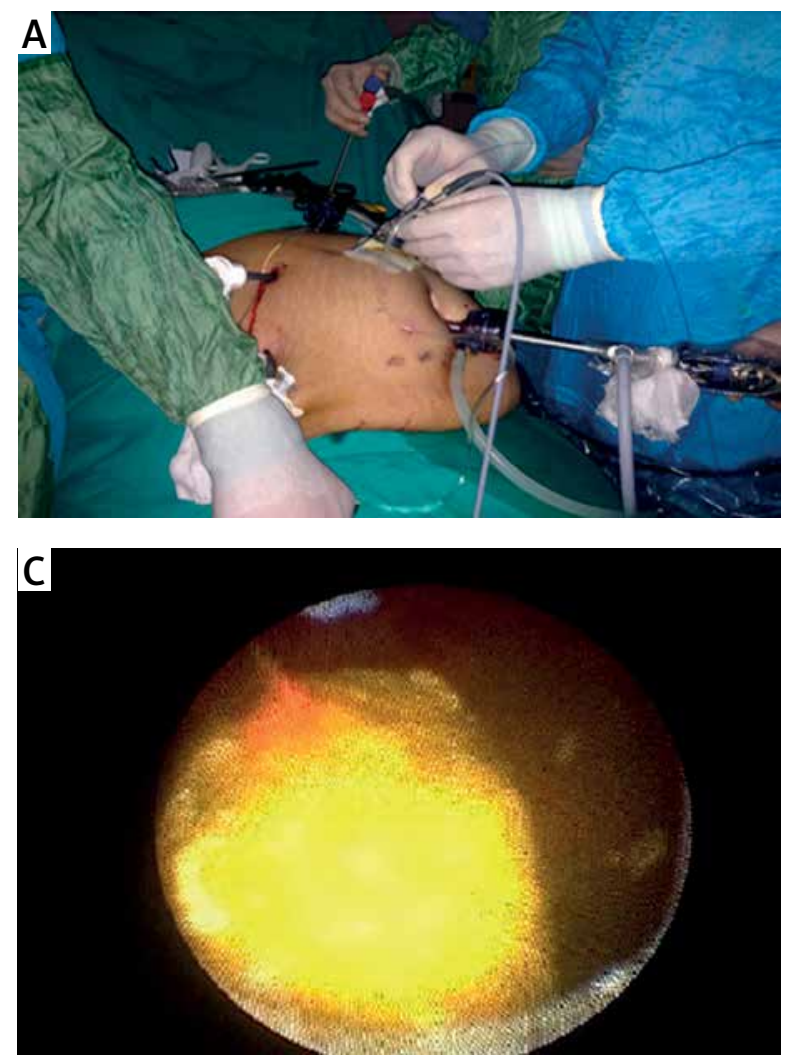

after the patient's discharge, pancreatic and biliary stents of the patient were endoscopically removed.

\section{Surgical technique}

The patient was placed in a supine position with her arms out. Trocars were placed in the standard form for laparoscopic cholecystectomy. After achieving pneumoperitoneum, a 12-mm umbilical camera trocar and two 12-mm working trocars was placed and then one 5-mm trocar was inserted for liver ecartation. Dissection of the dilated common hepatic duct was performed using the standard side-down technique. After identifying and dissecting the dilated common bile duct, the all-seeing needle was laparoscopically inserted in the pararectal area $2 \mathrm{~cm}$ above the umbilicus, under direct vision. A $4.8 \mathrm{G}$ needle was inserted in the middle of dilated common bile duct and then the lithotripsy procedure was performed on common hepatic bile duct stones near the common bile duct stent using a 200 micron laser fiber in $1 \mathrm{~J}$ at $5 \mathrm{~Hz}$ frequency with intermittent pump irrigation (Photos $3 \mathrm{~A}-\mathrm{C}$ ). Laser lithotripsy was continued to crush the stones at 1-2 $\mathrm{mm}$ size.

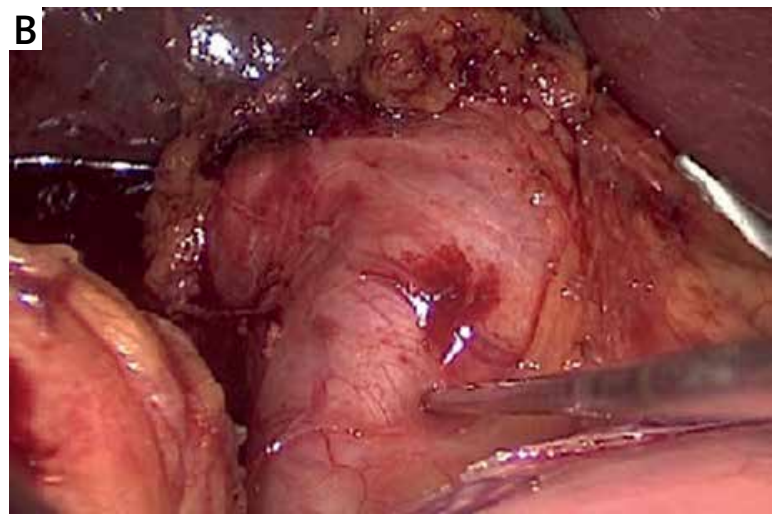

Photo 3. Intraoperative technical details of laparoscopy assisted micropercutaneous choledocholithotripsy: A - all-seeing needle was laparoscopically inserted in pararectal area above $2 \mathrm{~cm}$ umbilicus, B - $4.8 \mathrm{G}$ needle was implicated in the middle part of the dilatated choledoch; under direct vision, $\mathbf{C}$ - thripsy procedure was performed on common hepatic bile duct stone nearby choledoch stent by using 200 micron laser fiber with intermittant pump irrigation 
After the lithotripsy process was finished, cholangiography was performed through the all-seeing needle, and the common bile duct was demonstrated with free emptying of contrast into the duodenum. A 3/0 prolene stitch was used to close the implanted needle zone to prevent leakage of bile. The procedure was completed without inserting any duct tube such as a T-tube. The operation time was $70 \mathrm{~min}$ and estimated blood loss was $50 \mathrm{ml}$.

\section{Discussion}

In this report, the procedure of minimally invasive laparoscopy assisted micropercutaneous choledocholithotripsy with a holmium laser is presented for the first time in the literature. This technique can be preferred in complicated choledocholithiasis patients with a history of a failed ERCP secondary to a previous cholecystectomy. This technique provides significant advantages compared to other minimally invasive laparoscopic procedures including laparoscopic bile duct exploration and T-tube drainage, since these procedures are relatively invasive and complication-prone compared to the procedures described in our report. It can also reduce the length of hospital stay and management costs of the patients.

In patients with common bile duct stones, while open choledochotomy has previously been accepted as a standard treatment method in patients with failed ERCP, attention to these minimally invasive approaches has increased thanks to the development of laparoscopic and endoscopic surgical methods today. Laparoscopic common bile duct exploration is applied either as transcystic or direct choledochotomy, based on the size of the stone and the diameter of the cystic channel. In the presence of an indication for direct choledochotomy, the common bile duct can be primarily closed or decompression and T-tube drainage can be applied in order to obtain cholangiography during the postoperative period [5]. However, in patients to whom a T-tube is applied, complications such as pain in the drain area, leakage of bile, obstruction related to dislocation of the T-tube, continuous biliary fistula and secondary bile peritonitis related to dislocation of the T-tube can be seen due to this method at an approximate rate of $15 \%$ [6]. In addition to these complications, T-tube application increases the hospitalization period, surgery period and associated costs. As a result, there are some studies recommending primary repair after choledochotomy in order to reduce the hospitalization period, decrease the costs, reduce the complications related to the T-tube, and to enable the patients to return to their normal physical activities earlier [7]. In systematic studies including meta-analyses, it has been shown that there is no significant difference in terms of post-operative morbidity/mortality or biliary specific complications when laparoscopic bile duct exploration and primary repair are compared to the laparoscopic bile duct exploration and T-tube drainage method; and also that laparoscopic bile duct exploration and primary repair are advantageous in terms of hospitalization period and cost [8]. Alternative methods for the treatment of impacted common bile duct stones have been descried and laser lithotripsy is one of these methods. The holmium laser used in endourologic surgery is frequently used due to its penetration depth of 0.5-1 mm, low mucosal injury risk and high efficiency [9]. The holmium laser had successful results in bile ducts via flexible instruments and it can also efficiently fragment gallstones including cholesterol, calcium bilirubinate, and mixed content.

\section{Conclusions}

In this report, a novel procedure titled "laparoscopy-assisted micropercutaneous choledocholithotripsy with holmium laser lithotripsy" is presented for complicated choledocholithiasis cases with an unsuccessful ERCP attempt and previous cholecystectomy history. In this subset of patients, this technique has merits including several intraoperative and postoperative advantages and less possibility of potential complications, shorter hospital stay and reduced management costs.

\section{Conflict of interest}

The authors declare no conflict of interest.

\section{References}

1. Rosseland AR, Glomsaker TB. Asymptomatic common bile duct stones. Eur J Gastroenterol Hepatol 2000; 12: 1171-3.

2. Lyass S, Phillips EH. Laparoscopic transcystic duct common bile duct exploration. Surg Endosc 2006; 20: 441-5.

3. Orii K, Ozaki A, Takase Y, Iwasaki Y. Lithotomy of intrahepatic and choledochal stones with YAG laser. Surg Gynecol Obstet 1983; 156: 485-9.

4. Desai MR, Sharma R, Mishra S, et al. Single-step percutaneous nephrolithotomy (microperc): the initial clinical report. J Urol 2011; 186: 140-5. 
5. Isla AM, Griniatsos J, Karvounis E, Arbuckle JD. Advantages of laparoscopic stented choledochorrhaphy over T-tube placement. Br J Surg 2004; 91: 862-6.

6. Wills VL, Gibson K, Karihaloot C, Jorgensen JO. Complications of biliary T-tubes after choledochotomy. ANZ J Surg 2002; 72: 177-80.

7. Leida Z, Ping B, Shuguang W, Yu H. A randomized comparison of primary closure and T-tube drainage of the common bile duct after laparoscopic choledochotomy. Surg Endosc 2008; 22: 1595-600.

8. Podda M, Polignano FM, Luhmann A, et al. Systematic review with meta-analysis of studies comparing primary duct closure and T-tube drainage after laparoscopic common bile duct exploration for choledocholithiasis. Surg Endosc 2016; 30: 845-61.

9. Nazif OA, Teichman JM, Glickman RD, Welch AJ. Review of laser fibers: a practical guide for urologists. J Endourol 2004; 18: 818-29.

Received: 16.10 .2017 , accepted: 22.11.2017. 